\title{
Association of variants of transcription factor 7-like 2 (TCF 7L2) with susceptibility to type 2 diabetes in the Dutch Breda cohort
}

\author{
J. V. van Vliet-Ostaptchouk $\cdot$ R. Shiri-Sverdlov • \\ A. Zhernakova • E. Strengman • T. W. van Haeften • \\ M. H. Hofker • C. Wijmenga
}

Received: 4 July 2006 / Accepted: 5 September 2006 / Published online: 10 October 2006

(C) Springer-Verlag 2006

\begin{abstract}
Aim/hypothesis A strong association between susceptibility to type 2 diabetes and common variants of transcription factor 7-like 2 (TCF7L2), encoding an enteroendocrine transcription factor involved in glucose homeostasis, has been reported in three different populations (Iceland, Denmark and USA) by Grant et al. We aimed to replicate these findings in a Dutch cohort.

Methods We analysed the genotypes of two intronic single nucleotide polymorphisms (SNPs) in TCF7L2 gene in 502 unrelated type 2 diabetes patients and in a set of healthy controls $(n=920)$. The two SNPs showed almost complete linkage disequilibrium $\left(D^{\prime}=0.91\right)$.

Results We were able to replicate the previously reported association in our Breda cohort. The minor alleles of both variants were significantly over-represented in cases (odds ratio [OR] 1.29, 95\% CI 1.09-1.52, $p=3 \times 10^{-3}$ for rs12255372; OR 1.41, 95\% CI 1.19-1.66, $p=4.4 \times 10^{-5}$ for rs7903146). In addition, TCF7L2 haplotypes were analysed for association with the disease. The analysis of haplotypes did not reveal any strong association beyond that expected from analysing individual SNPs. The TT
\end{abstract}

M. H. Hofker and C. Wijmenga contributed equally.

J. V. van Vliet-Ostaptchouk · R. Shiri-Sverdlov •

M. H. Hofker $(\bowtie)$

Department of Molecular Genetics, Maastricht University,

Universiteitssingel 50 (UNS50/11), P.O. Box 616,

6200 MD Maastricht, the Netherlands

e-mail: m.hofker@gen.unimaas.nl

\footnotetext{
A. Zhernakova $\cdot$ E. Strengman - T. W. van Haeften · C. Wijmenga DBG-Department of Medical Genetics,

University Medical Center Utrecht,

Utrecht, the Netherlands
}

haplotype carrying the minor alleles was more frequent among cases (OR 1.38, $\left.p=2.7 \times 10^{-3}\right)$.

Conclusions/interpretation Our data strongly confirm that variants of the TCF $7 L 2$ gene contribute to the risk of type 2 diabetes. The population-attributable risk from this factor in the Dutch type 2 diabetes population is $10 \%$.

Keywords Association - Genetics · SNP. Susceptibility TCF4 · TCF7L2 - Type 2 diabetes · Variant
Abbreviations
LD linkage disequilibrium
OR odds ratio
SNP single nucleotide polymorphism
TCF7L2 transcription factor 7-like 2

\section{Introduction}

The TCF7L2 gene encodes for an enteroendocrine transcription factor that has a role in the Wnt signaling pathway, which is one of the key developmental and growth regulatory mechanisms of the cell. One of the peptide hormones produced by enteroendocrine cells and transcriptionally regulated by $T C F 7 L 2$ is glucagon-like peptide 1 (GLP-1). Hence, variants of the TCF7L2 gene could influence the susceptibility to type 2 diabetes by altering levels of the insulinotropic hormone GLP-1. In concert with insulin, GLP-1 exerts a critical effect on blood glucose homeostasis by stimulating early insulin production from the pancreatic beta cells [1].

Recently, Reynisdottir et al. [2] found suggestive linkage of type 2 diabetes to chromosome 10q in the Icelandic population. Fine-mapping with 228 microsatellite markers 
in Icelandic individuals with type 2 diabetes and controls throughout a $10.5 \mathrm{Mb}$ interval on $10 \mathrm{q}$ identified one microsatellite marker, DG10S478 in intron 3 of the TCF7L2 gene, as being strongly associated with type 2 diabetes $\left(p=2.1 \times 10^{-9}\right)$ [3]. This association was replicated in both a Danish cohort $\left(p=4.8 \times 10^{-3}\right)$ and a US cohort $\left(p=3.3 \times 10^{-9}\right)$. A combined analysis showed that individuals heterozygous for the risk allele $(38 \%$ of the population) have a modest risk of 1.45 of developing type 2 diabetes, whereas individuals homozygous for the risk allele ( $7 \%$ of the population) have a higher risk (2.41) of developing type 2 diabetes.

In the current study we examined whether variants of the TCF7L2 gene contribute to the risk of type 2 diabetes in a Dutch population, using 502 unrelated type 2 diabetes patients from the Breda cohort and a set of 920 healthy controls.

\section{Subjects and methods}

Breda cohort DNA was available from 502 subjects with type 2 diabetes from the Breda cohort (Table 1) [4]. All patients were diagnosed according to the WHO criteria (random plasma glucose levels $>11.1 \mathrm{mmol} / 1$ or a fasting plasma glucose level $>7.0 \mathrm{mmol} / \mathrm{l})$. The clinical characteristics of the patients $\left(\mathrm{HbA}_{1 \mathrm{c}}\right.$, plasma cholesterol, HDLcholesterol and triacyglycerol) were available, as well as the level of obesity in each individual given by the BMI (defined as weight [in $\mathrm{kg}$ ] divided by height [in $\mathrm{m}$ ] squared). The control cohort $(n=920)$ was comprised of healthy blood bank donors of white Dutch origin [5]. Informed written consent was obtained from all individuals. The Breda study was approved by the Medical Ethics Committee of the University Medical Center Utrecht.

Table 1 Descriptive statistics for study subjects from the type 2 diabetes Breda cohort and the control cohort

\begin{tabular}{|c|c|c|}
\hline Trait & Breda cohort & Control cohort \\
\hline$n$ (female/male) & $502\left(272 / 229^{a}\right)$ & $920\left(354 / 557^{\mathrm{b}}\right)$ \\
\hline Age-at-study (years) & $70.8 \pm 9.9$ & $47.8 \pm 12.7$ \\
\hline Age-at-diagnosis (years) & $63.0 \pm 11.3$ & - \\
\hline BMI $\left(\mathrm{kg} / \mathrm{m}^{2}\right)$ & $27.8 \pm 4.1$ & NA \\
\hline $\mathrm{HbA}_{1 \mathrm{c}}(\%)$ & $7.3 \pm 1.1$ & NA \\
\hline HDL-cholesterol (mmol/l) & $1.2 \pm 0.3$ & NA \\
\hline Total cholesterol (mmol/l) & $5.3 \pm 1.0$ & NA \\
\hline Triacylglycerol (mmol/l) & $1.9 \pm 1.0$ & NA \\
\hline
\end{tabular}

The data are presented as mean $\pm \mathrm{SD}$

$H b A_{1 c}$, haemoglobin $\mathrm{A}_{1 \mathrm{c}}$ (glucose bound to haemoglobin); NA, not available

${ }^{a}$ Not available for one subject; ${ }^{b}$ not available for nine subjects
Genotyping SNPs were genotyped using Taqman assay-ondemand (Applied Biosystems). Assays were performed according to the manufacturer's specifications (assays C_29347861_10, C_291484_20). The sequence information for all primers and probes is available upon request. The genotypes were analysed using a TaqMan 7900HT (Applied Biosystems, Nieuwerkerk a/d IJssel, the Netherlands). The DNA samples were processed in 384-well plates. Each plate contained eight negative controls and 16 genotyping controls (four duplicates of four different samples obtained from the Centre d'Etude du Polymorphisme Humain [CEPH]). The genotype success rates were $97.8 \%$ for rs 12255372 and $98.6 \%$ for rs 7903146 . There were no discordances in the genotypes of any of the CEPH samples. The controls were in agreement with HardyWeinberg equilibrium $\left(\chi^{2}=2.72, p=0.10\right.$ for rs 12255372 and $\chi^{2}=0.72, p=0.40$ for rs 7903146 ).

Statistical analysis The genotype frequencies were tested for Hardy-Weinberg equilibrium by $\chi^{2}$ analysis. Differences in allele, genotype and haplotype distribution in case and control subjects were tested for significance using a 95\% two-sided $\chi^{2}$ test. Odds ratios (ORs) and CIs were calculated using Woolf's method with Haldane's correction [6]. To test for association of genotypes and type 2 diabetes, genotype-based ORs with 95\% CIs were calculated using logistic regression model, where individuals homozygous for the common allele were the reference group. The association between genotypes of two SNPs and the cohort's clinical characteristics and BMI was determined using linear regression analysis, including age and sex as explanatory variables in the models. Haplotypes of two polymorphisms were estimated using the COCAPHASE package of UNPHASED program [7]. The $D^{\prime}$ and $r^{2}$ between the TCF7L2 SNPs were calculated with the same package. Population attributable risk (PAR) was calculated for rs7903146 using the corresponding genotype frequencies for this marker as explained by Greenland et al. [8]. Power calculation was completed using the UCLA's Department of Statistics Power Calculator (available at http://www.calculators.stat.ucla.edu/powercalc). All statistical analyses were performed using SPSS program, version 13.0 for Windows (SPSS, Chicago, IL, USA) unless otherwise stated.

\section{Results}

We genotyped two SNPs (rs12255372, rs7903146) that were shown to be most strongly associated with type 2 diabetes by Grant et al. [3]. Their study showed that the rs $12255372 * T$ allele is perfectly correlated with the DG10S478 risk 
allele. The rs 12255372 variant was successfully genotyped in 492 individuals from the Breda cohort and in 901 controls. The $T$ allele was significantly more frequent in patients than controls ( 34 vs $29 \%, p=0.003$ ), and the frequencies of the $G T$ and minor $T T$ genotypes were also increased in type 2 diabetes patients $(p=0.012)$ (Table 2$)$. However, this association was weaker than that observed with rs 7903146.

The variant rs7903146 in the TCF7L2 gene could be genotyped in 496 case and 907 control subjects. The $T$ allele was over-represented in the type 2 diabetes cohort compared with the control group ( 37 vs $29 \%, p=4.4 \times 10^{-5}$ ) (Table 2), which is compatible with the results of Grant et al. The $C T$ and $T T$ genotypes were more prevalent in the type 2 diabetes patients with a frequency of $45 \%$ and $15 \%$ in cases, respectively, compared with a frequency of $40 \%$ of $C T$ genotype and $9 \%$ of $T T$ genotype in control subject $(p=0.0003)$.

Risks were consistently higher for homozygous than for heterozygous persons for both SNPs (OR 1.53, 95\% CI $1.05-2.21$ and OR $1.35,95 \%$ CI $1.07-1.71$ for rs 12255372 and OR 1.96, 95\% CI $1.37-2.80$ and OR $1.37,95 \%$ CI 1.08-1.73 for rs7903146, respectively) (Table 2). Moreover, the test for trend showed even stronger association of the disease with an increased number of minor alleles, especially for rs7903146 $\left(p=6.6 \times 10^{-5}\right)$, suggesting an allele-dose effect.

The two SNPs were found to be in strong LD with each other $\left(D^{\prime}=0.91, r^{2}=0.76\right)$ in our control population. The $T T$ haplotype, which carries the two minor alleles of both SNPs, was more common among patients than controls (33 vs $26 \%, p=0.00027)$. As the haplotype does not perform better than the rs7903146 SNP located in intron 3 of TCF7L2, this SNP can be used as a marker for type 2 diabetes risk. The allele frequency data for rs7903146 revealed that individuals carrying the $T$ allele $(37 \%$ frequency in patients compared with $29 \%$ in control subjects) have a modest but significantly higher risk of developing type 2 diabetes (OR 1.41, 95\% CI 1.19-1.66), with a population attributable risk of $10 \%(95 \%$ CI 0.054 0.155). These results strongly suggest that TCF7L2 is associated with type 2 diabetes in the Dutch Breda cohort.

\section{Discussion}

Recently Grant et al. [3] demonstrated a convincing association between susceptibility to type 2 diabetes and common variants in TCF $7 L 2$. In order to replicate these findings, two TCF7L2 SNPs (rs12255372 and rs7903146) were genotyped in the Dutch Breda cohort and a control group. Our study had power of $80 \%$ at $p=0.05$ to detect effects with an OR $>1.39$ for the examined variants. Consistent with the observation of Grant et al., we found the minor alleles of both variants were significantly overrepresented in Dutch type 2 diabetes patients. The frequency of heterozygous and homozygous carriers of the at-risk alleles, compared with non-carriers, was also increased in type 2 diabetes patients. In line with Grant et al., we also revealed a negative, borderline-significant association with BMI (data not shown), suggesting that BMI does not influence the risk variants.

Despite serious efforts to identify genetic variants that predispose to common forms of type 2 diabetes, till now only a few genes, such as KCNJ11, PPARG and HNF4A have been reproducibly associated with this complex disease in a variety of large-scale studies performed in the different populations $[9,10]$. Our replication study shows that the TCF7L2 gene is an important candidate gene contributing to susceptibility to type 2 diabetes. Since the most strongly associated SNP is within an intron of the TCF7L2 gene, this SNP is not likely to be the true disease variant. However, the intronic variants may affect the level of gene expression by altering, for example, transcription,
Table 2 Comparison of genotype and allele frequencies in the Breda cohort and control subjects

$T 2 D$, type 2 diabetes

\begin{tabular}{|c|c|c|c|c|c|}
\hline Genotype & T2D patients $(n=502)$ & Control subjects $(n=920)$ & OR & $95 \% \mathrm{CI}$ & $p$ value \\
\hline \multicolumn{6}{|l|}{ rs 12255372} \\
\hline GG $(\%)$ & $216(44)$ & $469(52)$ & - & - & - \\
\hline GT $(\%)$ & $217(44)$ & 348 (39) & 1.35 & $1.07-1.71$ & 0.01 \\
\hline TT (\%) & $59(12)$ & $84(9)$ & 1.53 & $1.05-2.21$ & 0.03 \\
\hline$p$ value genotype & & & & & 0.012 \\
\hline G allele $(\%)$ & $649(66)$ & $1286(71)$ & & & \\
\hline $\mathrm{T}$ allele $(\%)$ & $335(34)$ & $516(29)$ & 1.29 & $1.09-1.52$ & 0.003 \\
\hline \multicolumn{6}{|l|}{ rs7903146 } \\
\hline $\mathrm{CC}(\%)$ & $203(41)$ & $459(51)$ & - & - & - \\
\hline CT $(\%)$ & $221(45)$ & $365(40)$ & 1.37 & $1.08-1.73$ & 0.009 \\
\hline TT $(\%)$ & $72(15)$ & $83(9)$ & 1.96 & $1.37-2.80$ & 0.0002 \\
\hline$p$ value genotype & & & & & 0.0003 \\
\hline $\mathrm{C}$ allele $(\%)$ & $627(63)$ & $1283(71)$ & & & \\
\hline $\mathrm{T}$ allele $(\%)$ & $365(37)$ & $531(29)$ & 1.41 & $1.19-1.66$ & 0.00004 \\
\hline
\end{tabular}


splicing or message stability. Hence, full sequence analysis of the gene and a comparison of the allelic architecture among different type 2 diabetes populations are needed to identify the causative variant. The TCF $2 L 2$ gene probably contributes to the complex nature of type 2 diabetes by affecting the blood glucose homeostasis, since TCF $7 L 2$ is known to control the activity of a number of genes, including the proglucagon gene that encodes the insulinotropic hormone GLP-1. GLP-1, along with insulin, plays a role in blood glucose homeostasis, and GLP-1 therapies for type 2 diabetes are currently in clinical development. As long as the exact role of these gene polymorphisms remains unclear, it is difficult to assess its functional role in the development of type 2 diabetes.

It will be of great interest to learn the molecular mechanisms of action of the TCF7L2 gene in the pathogenesis of type 2 diabetes and to identify potential targets for drugs that could be used for treatment and prevention of the disease due to the gene variants. It is tempting to speculate on a future genetic test that could identify the individuals carrying the at-risk TCF7L2 gene variants, so that they could be offered a preventive healthcare programme (special diet and exercises).

In conclusion, we have confirmed that variants of the TCF7L2 gene modulate susceptibility to type 2 diabetes in a Dutch population.

Acknowledgements We thank the Dutch Diabetes Foundation, the European Vascular Genomics Network and SenterNovem (IOP Genomics IGE05012) for financial support. We would like to thank Jackie Senior for critically reading the manuscript and Behrooz Alizadeh and N. Charlotte Onland-Moret for statistical advice.
Duality of interest The authors confirm that there is no duality of interest.

\section{References}

1. Yi F, Brubaker PL, Jin T (2005) TCF-4 mediates cell type-specific regulation of proglucagon gene expression by beta-catenin and glycogen synthase kinase-3beta. J Biol Chem 280:1457-1464

2. Reynisdottir I, Thorleifsson G, Benediktsson R et al (2003) Localization of a susceptibility gene for type 2 diabetes to chromosome 5q34-q35.2. Am J Hum Genet 73 323-335

3. Grant SF, Thorleifsson G, Reynisdottir I et al (2006) Variant of transcription factor 7-like 2 (TCF7L2) gene confers risk of type 2 diabetes. Nat Genet 38:320-323

4. Van Tilburg J, Sandkuijl LA, Strengman E et al (2003) A genomewide scan in type 2 diabetes mellitus provides independent replication of a susceptibility locus on $18 \mathrm{p} 11$ and suggests existence of novel loci on $2 \mathrm{q} 12$ and $19 \mathrm{q} 13$. J Clin Endocrin Metab 88:2223-2230

5. Monsuur AJ, de Bakker PI, Alizadeh BZ et al (2005) Myosin IXB variant increases the risk of celiac disease and points toward a primary intestinal barrier defect. Nat Genet 37(12):1341-1344

6. Haldane JB (1956) The estimation and significance of the logarithm of a ratio of frequencies. Ann Hum Genet 20:309-311

7. Dudbridge F (2003) Pedigree disequilibrium tests for multilocus haplotypes. Genet Epidemiol 25(2):115-121

8. Greenland S, Rothman RK (1998) Measures of effect and measures of association. In: Greenland S, Rothman RK (eds) Modern epidemiology, 2nd edn. Lippincott-Raven, Philadelphia, pp 47-67

9. O'Rahilly S, Barroso I, Wareham NJ (2005) Genetic factors in type 2 diabetes: the end of the beginning? Science 307 (5708):370-373

10. Barroso I (2005) Genetics of type 2 diabetes. Diabet Med 22 (5):517-535 